Pacific Journal of Mathematics

VARIETIES OF IMPLICATIVE SEMILATTICES

VAR CHARLES NEMITZ AND THOMAS PAUL WHALE Y 


\title{
VARIETIES OF IMPLICATIVE SEMILATTICES
}

\author{
W. Nemitz AND T. Whaley
}

The main purpose of this paper is to investigate properties of the lattice of subvarieties of the variety of implicative semilattices. Also the distinct compositions of the operators of taking homomorphic images, subalgebras, and products of classes of implicative semilattices are determined.

A class $K$ of similar universal algebras [2, pp. 33-34] is called a variety provided $K$ consists of all the algebras which satisfy some set of identities. If $K$ is a variety and $K^{\prime} \subseteq K$, then $K^{\prime}$ is called a subvariety of $K$ provided $K^{\prime}$ is itself a variety. The subvarieties of a given variety form a lattice when ordered by inclusion. A basic theorem of Birkhoff [1] states that a class of algebras $K$ is a variety if and only if $K$ is closed under the taking of homomorphic images, subalgebras, and direct products.

An implicative semilattice is an algebra $\langle L ; \wedge, *\rangle$ where $\langle L ; \wedge\rangle$ is a semilattice, and $*$ is a binary operation such that $x \wedge y \leqq z$ if and only if $x \leqq y * z$ (here $w \leqq u$ means $w \wedge u=w$ ). Every implicative semilattice has a largest element which we denote by 1 . Monteiro [4] has given a set of equational axioms for implicative semilattices thus showing that the class of implicative semilattices is a variety. In this paper we consider the lattice of subvarieties of this variety. We shall denote the variety of all implicative semilattices by $I$.

An ideal $K$ of an implicative semilattice $L$ is a subset of $L$ such that $x \in K$ whenever $x \leqq y \in K$. A filter $J$ of $L$ is a subset of $L$ such that $x \wedge y, z \in J$ whenever $x, y \in J, z \in L$ and $x \leqq z$. It is shown in [5] that filters are related to homomorphisms for implicative semilattices in the same way they are for boolean algebras. In particular, if by the kernel of a homomorphism we mean the pre-image of the greatest element of the range, then the kernel of any homomorphism is a filter, every filter is the kernel of a homomorphism, and the congruence relation and quotient algebra determined by the homomorphism are also determined by the kernel of the homomorphism. Also, in [6] it is shown that the lattice of filters and therefore the lattice of congruence relations of an implicative semi-lattice is distributive. Thus we are able to make use of the results and techniques of Jónsson [3].

In $\S 2$ we determine several elementary properties of subdirectly irreducible implicative semilattices. Here it is also shown that the variety of implicative semilattices is generated by its finite members, 
covers no subvariety, and is not the lattice join of any two proper subvarieties. In $\S 3$ we determine the varieties generated by chains and give identities for these. In $\S 4$ we introduce the notion of sublength for implicative semilattices and show that this is a variety property. The varieties of sublength three are determined by their generators.

For a class $K$ of algebras $H(K), S(K), P(K)$ denote respectively the class of all homomorphic images of elements of $K$, the class of all subalgebras of elements of $K$, and the class of all direct products of elements of $K$. Pigozzi [8] has determined all direct compositions of the operations $H, S$, and $P$ for arbitrary algebras. In the final section we consider this problem when restricted to classes of implicative semilattices.

For the basic arithmetic of implicative semilattices, the reader is referred to [5].

2. Subdirectly irreducible implicative semilattices. Since a subdirectly irreducible algebra is one with a smallest proper congruence relation, we see that a subdirectly irreducible implicative semilattice is one with a smallest proper filter, thus one with a unique dual atom which dominates all elements other than 1 . If we let $K^{e}$ denote the smallest variety containing the class of algebras $K$, then $K^{e}=H S P(K)$. Since a variety is determined by its subdirectly irreducible members, for a given class $K$ of algebras, it is helpful to know the subdirectly irreducible members of $K^{e}$. It follows from [3, Cor. 3.4] that if $K$ is a finite set of finite implicative semilattices, then every subdirectly irreducible member $L$ of $K^{e}$ is in $H S(K)$. The following lemma shows that $L$ is actually in $S(K)$.

LeMma 2.1. If $L$ is an implicative semilattice every filter of which is principal, then any homomorphic image of $L$ is isomorphic to a subalgebra of $L$.

Proof. Suppose $\varphi: L \rightarrow L^{\prime}$ is a homomorphism. The kernel of $\varphi$ is principal, say generated by $a$. It is easy to check that the mapping $\psi: L \rightarrow L$ which takes $x$ to $a * x$ is a homomorphism with the same kernel as $\varphi$. Thus $\psi(L) \cong \varphi(L)$.

If $L$ is any implicative semilattice, we let $\hat{L}$ be the result of adjoining an element $u$ to $L$ in such a way that $x<u<1$ for each $x \in L \sim\{1\}$. The operations of $L$ extend those of $\hat{L}$ and satisfy the formulas

$$
1 * u=u, u * 1=1
$$

and 


$$
x * u=1, u * x=x \text { for all } x \in L \sim\{1\} .
$$

Clearly $\hat{L}$ is subdirectly irreducible and $L \leqq_{s} \hat{L}$ ( $L$ is a subalgebra of $\hat{L})$. Also note that every subdirectly irreducible implicative semilattice can be so obtained. It is easy to check that the mapping $\varphi$ defined by $\varphi(x)=u * x$ is a homomorphism of $\hat{L}$ onto $L$. Thus we get the following lemma.

LEMMA 2.2. Every implicative semilattice is both a subalgebra and a homomorphic image of the same subdirectly irreducible implicative semilattice.

By [3, Lemma 4.1] we see that if $V_{1}$ and $V_{2}$ are varieties of implicative semilattices and if $L$ is a subdirectly irreducible member of $\left(V_{1} \cup V_{2}\right)^{e}$, then $L \in V_{1}$ or $L \in V_{2}$. Using Lemma 2.2 and a proof as in [3, Thm. 5.4] we get the following corollary.

COROLlaRy 2.3. If $V_{1}$ and $V_{2}$ are proper subvarieties of $I$, then so is the lattice join of $V_{1}$ and $V_{2}$.

The following lemma shows that $I$ is generated by its finite members.

Lemma 2.4. Let $L$ be an implicative semilattice, and let $S$ be a finite, nonempty subset of $L$ which is closed under $\wedge$. Then there is a finite implicative semilattice $L^{\prime}$ and a one-to-one, order preserving mapping $\varphi$ of $S$ into $L^{\prime}$ such that

(i ) $\varphi(x \wedge y)=\varphi(x) \wedge \varphi(y)$ if $x, y \in S$,

(ii) $\varphi(x * y)=\varphi(x) * \varphi(y)$. If $x, y, x * y \in S$.

Proof. Let $L^{\prime}$ be the set of all ideals of $S$ ordered by inclusion. Clearly $L^{\prime}$ is closed under intersection. For $x \in S$, let $(x)=\{y \in S: y \leqq x\}$ so $(x) \in L^{\prime}$. For $A_{1}, A_{2} \in L^{\prime}$, let

$$
A_{1} \circ A_{2}=\left\{x \in S:(x) \cap A_{1} \subseteq A_{2}\right\} .
$$

Obviously $A_{1} \circ A_{2} \in L^{\prime}$. If $x \in A_{1} \cap\left(A_{1} \circ A_{2}\right)$, then $(x)=(x) \cap A_{1} \subseteq A_{2}$ so $x \in A_{2}$. Thus $A_{1} \cap\left(A_{1} \circ A_{2}\right) \leqq A_{2}$. If $A \in L^{\prime}, A_{1} \cap A \leqq A_{2}$, and $x \in A$, then $(x) \cap A_{1} \subseteq A_{2}$ so $x \in A_{1} \circ A_{2}$. Hence $A \subseteq A_{1} \circ A_{2}$. Therefore, $\left\langle L^{\prime} ; \cap, \circ\right\rangle$ is an implicative semilattice.

Now let $\varphi: S \rightarrow L^{\prime}$ by $\varphi(x)=(x)$. This mapping is clearly one-toone and order preserving. Also it is clear that $\varphi(x \wedge y)=\varphi(x) \cap \varphi(y)$ if $x, y \in S$. Hence $\varphi(x) \cap \varphi(x * y) \subseteq \varphi(y)$, so $\varphi(x * y) \subseteq \varphi(x) \circ \varphi(y)$. If $w \in \varphi(x) \circ \varphi(y)$, then $(w) \cap(x) \leqq(y)$ so $w \wedge x \leqq y$. Thus $w \leqq x * y$ and $w \in(x * y)=\varphi(x * y)$. Hence $\varphi(x * y)=\varphi(x) \circ \varphi(y)$ and the proof is complete. 
THEOREM 2.5. I is the smallest variety of implicative semilattices which contains all of the finite implicative semilattices.

Proof. From Lemma 2.4 it follows that any identity which does not hold in every implicative semilattice fails in some finite implicative semilattice.

COROLlaRY 2.6. There is no variety $V$ which $I$ covers in the lattice of subvarieties of $I$.

Proof. Same as [3, Cor. 5.5].

LEMma 2.6. If $K$ is a class of similar algebras and if $A$ is a subdirectly irreducible member of $S P(K)$, then $A \in S(K)$.

Proof. Suppose $A \leqq_{s} \prod_{i \in I} A_{i}$ where each $A_{i} \in K$. Let $\varphi_{i}$ be the projection of $A$ into $A_{i}$. Then $A$ is a subdirect product of $\left\{\varphi_{i}(A): i \in I\right\}$. Thus one of the projections must be a monomorphism.

3. Varieties generated by chains. ${ }^{1}$ From the remarks of the preceeding section it is clear that any chain with a largest element and a dual atom is a subdirectly irreducible implicative semilattice. For $n \in \omega \sim\{0\}$, let $\mathscr{C}_{n}$ denote the variety generated by an $n$-element chain. Let $\mathscr{C}$ denote the variety generated by all finite chains; $\mathscr{C}=\mathrm{V} \mathscr{C}_{n}$. From Lemma 2.1 of the preceeding section it is clear that for $m<n$ we have $\mathscr{C}_{m} \subset \mathscr{C}_{n}$. In fact $\mathscr{C}_{n+1}$ covers $\mathscr{C}_{n}$ in the lattice of subvarieties.

Theorem 3.1. If $C$ is any infinite chain with 1 , then $\mathscr{C}$ is the variety generated by $C$. Hence $\mathscr{C}$ is the variety generated by the class of all chains.

Proof. Since $C$ contains each finite chain as a subalgebra we clearly have $\mathscr{C} \subseteq\{C\}^{e}$. The reverse inclusion is obtained by a standard argument. If an identity fails in $C$ it fails in a finite subchain of $C$. This subchain together with 1 is a subalgebra of $C$ in which the identity fails. Thus $C$ satisfies every identity which holds in each finite chain, so $C \in \mathscr{C}$.

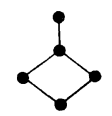

FIGURE 1

Notation. Let $D^{\prime}$ denote the implicative semilattice of Figure 1.

1 T. Katrinak has obtained results similar to some of those in this and the next sections. 
LEMMA 3.2. If $L$ is a subdirectly irreducible implicative semilattice and $L$ is not a chain, then $D^{1} \leqq L$.

Proof. Let $u$ be the dual atom of $L$ and let $a, b$ be elements of $L$ which are not related. It is straightforward to check that

$$
\{1, u, a * b, b * a,(a * b) \wedge(b * a)\}
$$

is a subalgebra of $L$. To see that these elements are distinct it is enough to show that $a * b$ and $b * a$ are not related. But if $a * b \leqq$ $b * a$, then

$$
b=b \wedge b \leqq b \wedge(a * b) \leqq b \wedge(b * a) \leqq a
$$

a contradiction.

In [7] the operation of pseudo-join for implicative semilattices was introduced. This operation is given by

$$
a+b=((a * b) * b) \wedge((b * a) * a) .
$$

It was shown that this operation is a lattice join precisely when the operation is associative. Semi-Boolean lattices are those implicative semilattices in which the pseudo-join is associative.

\section{THEOREM 3.3. $\mathscr{C}$ is the class of semi-Boolean lattices.}

Proof. It is clear that in any chain $a+b$ is the larger of $a$ and $b$. Thus any chain is a semi-Boolean lattice. It then suffices to show that any subdirectly irreducible implicative semilattice which is not a chain is not semi-Boolean. For this it is enough to show that $D^{1}$ is not semi-Boolean. If $a$ and $b$ are the two unrelated elements of $D^{1}$ then $a+b=1$ which is not the least upper bound of $a$ and $b$.

Theorem 3.4. For $n=1,2, \cdots$, the identity

$$
I_{n}:\left(x_{1} * x_{2}\right)+\left(x_{2} * x_{3}\right)+\cdots+\left(x_{n} * x_{n+1}\right)=1
$$

holds in $C_{n}$ but fails in $C_{n+1}$.

Proof. In the $n$-element chain, for any elements $a_{1}, a_{2}, \cdots, a_{n+1}$, we must have $a_{i} \leqq a_{i+1}$ for some $i$ so $a_{i} * a_{i+1}=1$.

In the $n+1$ element chain, we have $a_{1}>a_{2}>\cdots>a_{n+1}$ so

$$
\begin{aligned}
\left(a_{1} * a_{2}\right)+\left(a_{2} * a_{3}\right)+\cdots+\left(a_{n} * a_{n+1}\right) & =a_{2}+a_{3}+\cdots+a_{n+1} \\
& =a_{2} .
\end{aligned}
$$

CoRollary 3.5. $\mathscr{C}_{n}$ is characterized by $I_{n}$ together with the associative law for + . 
Proof. Let $\dot{V}$ be the variety defined by $I_{n}$ and the associative law for + . By Theorems 3.3 and 3.5 we see that $V$ is a proper subvariety of $\mathscr{C}$ which contains $\mathscr{C}_{n}$. Since $D^{1}$ is not semi-Boolean, we see by Lemma 3.2 that the only subdirectly irreducible members of $\mathscr{C}$ are chains. Thus any subvariety of $\mathscr{C}$ which properly contains $\mathscr{C}_{n}$ must contain $C_{n+1}$. Since $V$ does not contain $C_{n+1}$ we have $V=\mathscr{C}_{n}$.

\section{Semilattices of finite sublength.}

Definition 4.1. For $n=1,2, \cdots$ an implicative semilattice $L$ is said to have sublength $n$ provided $C_{n}$ is a subalgebra of $L$ but $C_{n+1}$ is not. We let $\overline{\mathscr{C}}_{n}$ denote the class of implicative semilattice having sublength less than or equal to $n$.

LEMma 4.2. If $f: L \rightarrow C_{n}$ is an epimorphism, then $L$ has a subalgebra isomorphic to $C_{n}$ which is mapped onto $C_{n}$ by $f$.

Proof. The proof is by induction on $n$. The case $n=1$ is clear. Suppose the result is true for $n=k$ and that $f: L \rightarrow C_{k+1}$. Let $C_{k+1}=$ $\left\{1=a_{1}, a_{2}, \cdots, a_{k}, a_{k+1}\right\}$ where $a_{1}>a_{2}>\cdots>a_{k}>a_{k+1}$. Let

$$
\left\{1=x_{1}, x_{2}, \cdots, x_{k}, x_{k+1}\right\} \subseteq L
$$

such that $f\left(x_{i}\right)=a_{i}$ for $i=1, \cdots, k+1$. We may assume that $x_{1}>$ $x_{2}>\cdots>x_{k}>x_{k+1}$ since $x_{1}>x_{1} \wedge x_{2}>\cdots>x_{1} \wedge \cdots \wedge x_{k+1}$ and

$$
f\left(x_{1} \wedge \cdots \wedge x_{j}\right)=a_{j} .
$$

If we restrict $f$ to the principal filter generated by $x_{k}$ and apply the induction hypothesis, we may assume that $x_{i} * x_{j}=x_{j}$ if $i<j<k$. We focus our attention on the subalgebra of $L$ generated by

$$
\left\{x_{1}, \cdots, x_{k+1}\right\} \text {. }
$$

Clearly $x_{k+1}$ is the least element of this subalgebra so we denote it by 0 . Also we denote $a_{k+1}$ by 0 . For $2 \leqq j \leqq k$ let $y_{j}=x_{k}^{* *} * x_{j}$ where $x^{*}=x * 0$. We now claim that $\left\{1, y_{2}, \cdots, y_{k}, 0\right\}$ is the subalgebra of $L$ which we desire.

It is clear that $1 \geqq y_{2} \geqq \cdots \geqq y_{k} \geqq 0$. However,

$$
\begin{aligned}
f\left(y_{i}\right) & =f\left(x_{k}^{* *} * x_{i}\right) \\
& =f\left(x_{k}^{* *}\right) * a_{i} \\
& =\left[\left(a_{k} * 0\right) * 0\right] * a_{i} \\
& =(0 * 0) * a_{i} \\
& =a_{i} .
\end{aligned}
$$


Thus we have $1>y_{2}>\cdots>y_{k}>0$ and $f\left(y_{i}\right)=a_{i}$.

To check that $\left\{1, y_{2}, \cdots, y_{k}, 0\right\}$ is a subalgebra of $L$, we only need to show that $y_{j} * 0=0$ and that $y_{i} * y_{j}=y_{j}$ if $1<i<j \leqq k$. Since $y_{j}=x_{k}^{* *} * x_{j} \geqq x_{j} \geqq x_{k}$, we have $y_{j} * 0 \leqq x_{k} * 0=x_{k}^{*}$. Also,

$$
y_{i}=x_{k}^{* *} * x_{i} \geqq x_{k}^{* *} * 0=x_{k}^{* * *}=x_{k}^{*} .
$$

Thus $y_{i} * 0 \leqq x_{k}^{*} * 0=x_{k}^{* *}$. Hence $y_{i} * 0 \leqq x_{k}^{*} \wedge x_{k}^{* *}=0$ so $y_{i} * 0=0$. Furthermore,

$$
\begin{aligned}
y_{i} * y_{j} & =\left(x_{k}^{* *} * x_{i}\right) *\left(x_{k}^{* *} * x_{j}\right) \\
& =x_{k}^{* * *} *\left(x_{i} * x_{j}\right) \\
& =x_{k}^{* * *} * x_{j} \\
& =y_{j}
\end{aligned}
$$

and the proof is complete.

Theorem 4.3. For $n=1,2, \cdots, \overline{\mathscr{C}}_{n}$ is a variety.

Proof. Clearly $\overline{\mathscr{C}}_{n}$ is closed under the taking of subalgebras, by Lemma 2.6 it is closed under products, and by Lemma 4.2 it is closed under homomorphisms.

Corollary 4.4. Let $I$ be the variety of all implicative semilattices. Then $\mathrm{V}_{n \in \omega} \overline{\bar{C}_{n}^{\prime}}=I$.

Proof. This is clear since $\mathbf{V}_{n \in \omega} \overline{\mathscr{C}}_{n}$ contains all of the finite implicative semilattices.

TheoRem 4.5. For $n=1,2, \cdots \overline{\mathscr{C}}_{n}$ is characterized by $I_{n}$ (with all association in $I_{n}$ being to the right).

Proof. Let $V_{n}$ be the variety determined by $I_{n}$. We show by induction on $n$ that $V_{n}=\overline{\mathscr{C}}_{n}$. If $L \notin \overline{\mathscr{C}}_{n}$, then $L$ contains $C_{n+1}$ as a subalgebra; so by Theorem $3.4 I_{n}$ fails in $L$. Thus we see that $V_{n} \subseteq \overline{\mathscr{C}}_{n}$. Hence we only need to show that the subdirectly irreducible members of $\overline{\mathscr{C}}_{n}$ are in $V_{n}$. The case $n=1$ is clear since $\overline{\mathscr{C}}_{1}$ consists only of one element algebras.

Now assume that $I_{k}$ characterizes $\overline{\mathscr{C}}_{k}$ and that $\hat{L}$ is a subdirectly irreducible member of $\overline{\mathscr{C}}_{k+1}$. Suppose $a_{1}, a_{2}, \cdots, a_{k+2} \in \hat{L}$ and that

$$
\left(a_{1} * a_{2}\right)+\left(\left(a_{2} * a_{3}\right)+\cdots+\left(\left(a_{k} * a_{k+1}\right)+\left(a_{k+1} * a_{k+2}\right)\right) \cdots\right)<1 .
$$

Then we must have $a_{1} * a_{2}, a_{2} * a_{3}, \cdots, a_{k} * a_{k+2}<1$. From this we get $a_{2}, a_{3}, \cdots, a_{k+2}<1$. Also we have 


$$
\left(a_{2} * a_{3}\right)+\left(\left(a_{3} * a_{4}\right)+\cdots+\left(\left(a_{k} * a_{k+1}\right)+\left(a_{k+1} * a_{k+2}\right)\right)<1 .\right.
$$

By the induction hypothesis we have $I_{k}$ holding in $L$ since $L \in \overline{\mathscr{C}}_{k}$. Thus one of $a_{2}, a_{3}, \cdots, a_{k+2}$ must be the dual atom of $\hat{L}$, call it $u$. Now since $a_{2} \leqq u, a_{2} * a_{3} \leqq u$ we must have $a_{3}<u$. Similarly $a_{i}<u$ for $i=3,4, \cdots, k+2$. Hence $a_{2}=u$. Then we get

$$
\begin{aligned}
1 & \left.>\left(a_{2} * a_{3}\right)+\cdots+\left(\left(a_{k} * a_{k+1}\right)+\left(a_{k+1} * a_{k+2}\right)\right) \cdots\right) . \\
& \left.=\left(u * a_{3}\right)+\cdots+\left(\left(a_{k} * a_{k+1}\right)+\left(a_{k+1} * a_{k+2}\right)\right) \cdots\right) . \\
& \left.=\left(1 * a_{3}\right)+\cdots+\left(\left(a_{k} * a_{k+1}\right)+\left(a_{k+1} * a_{k+2}\right)\right) \cdots\right) .
\end{aligned}
$$

But this contradicts the assumption that $I_{k}$ holds in $L$, and the proof is complete.

LeMma 4.6. Let $\hat{L}$ be any subdirectly irreducible member of $\overline{\mathscr{C}}_{3}$ which has a least element 0 . Then $L$ is a Boolean algebra.

Proof. If $x \in L \sim\{1\}$ we can not have $x * 0=0$ or else $\{1, u, x, 0\}$ is a four element subchain of $L$. Using Theorems 4.3 and 4.4 of [5] we see that $L$ is a Boolean algebra. (Note that $\hat{L} \in \overline{\mathscr{C}}_{n+1}$ implies that $\left.L \in \overline{\mathscr{C}}_{n}\right)$.

CoROLlary 4.7. $\overline{\mathscr{C}}_{3}$ is generated by its finite members.

Proof. Suppose not. Let $V$ be the variety generated by the finite members of $\overline{\mathscr{C}}_{3}$. Then there is a subdirectly irreducible member of $\overline{\mathscr{C}}_{3} \sim V$, say $L$. Thus there is some identity which holds in $V$ but fails in $L$. The failure of $L$ to satisfy this identity depends only on a finite number of elements of $L$. Thus we may assume $L$ is finitely generated. However, this would imply that $L$ has a least member giving us $L$ a finite Boolean algebra.

CoROLlaRY 4.8. Let $\mathscr{B}_{m}$ denote the variety generated by $B_{m}$ where $B_{m}$ is the $2^{m}$-element Boolean algebra. If $m<n$, then $\mathscr{B}_{m} \subset \mathscr{B}_{n}$. Also we have $\mathrm{V}_{n \in \omega} \cdot \mathscr{P}_{n}=\overline{\mathscr{C}}_{3}$.

COROLLARY 4.9 If $B$ is any infinite Boolean algebra, then $\hat{B}$ generates $\overline{\mathscr{C}}_{3}$.

5. H, S, P for implicative semilattices.

DEFINITION 5.1. If $Q$ and $Q^{\prime}$ are two compositions of the operators $H, S, P$, we let $Q \leqq Q^{\prime}$ mean that $Q(K) \leqq Q^{\prime}(K)$ for every class $K$ of 
implicative semilattices. Also $Q<Q^{\prime}$ mean $Q \leqq Q^{\prime}$, but $Q(K) \subset Q^{\prime}(K)$ for some class $K$.

It is well known that for any class $K$ of algebras we have $S H(K) \subseteq$ $H S(K), P H(K) \subseteq H P(K)$, and $P S(K) \subseteq S P(K)$. Pigozzi [8] has shown using these inequalities that any composition of $H, S, P$ is equal to one of the compositions without a repeated term or to either SHPS or SPHS. He has also shown that no further reductions are possible. For implicative semilattices, we shall show that $H S=S H$ and that the reductions which follow from this equality are the only one possible for implicative semilattices.

LEMMA 5.1. If $L$ and $L^{\prime}$ are implicative semilattices and if $L^{\prime}$ is a homomorphic image of a subalgebra of $L$, then $L^{\prime}$ is a subalgebra of a homomorphic image of $L$.

Proof. Suppose $L^{\prime \prime} \leqq{ }_{s} L$ and $f: L^{\prime \prime} \rightarrow L^{\prime}$ is an epimorphism. Let $J=\operatorname{ker}(f)$. Then $L^{\prime} \cong L^{\prime \prime} / J$. Let $K$ be the filter of $L$ generated by $J$. It is clear that $K$ restricted to $L^{\prime \prime}$ is $J$ so $L^{\prime} \cong L^{\prime \prime} / J \cong L^{\prime \prime} /\left(K \cap L^{\prime \prime}\right) \leqq_{s} L / K$.

TheOREM 5.2. For implicative semilattices,

$$
H S=S H, H S P=S H P, P H S=P S H, S H P S=H S P
$$

and $S P H S=S P H$. No further reduction is possible.

Proof. All of the equalities follow easily from the first which is immediate from Lemma 5.1.

To establish that no further reduction is possible we show that

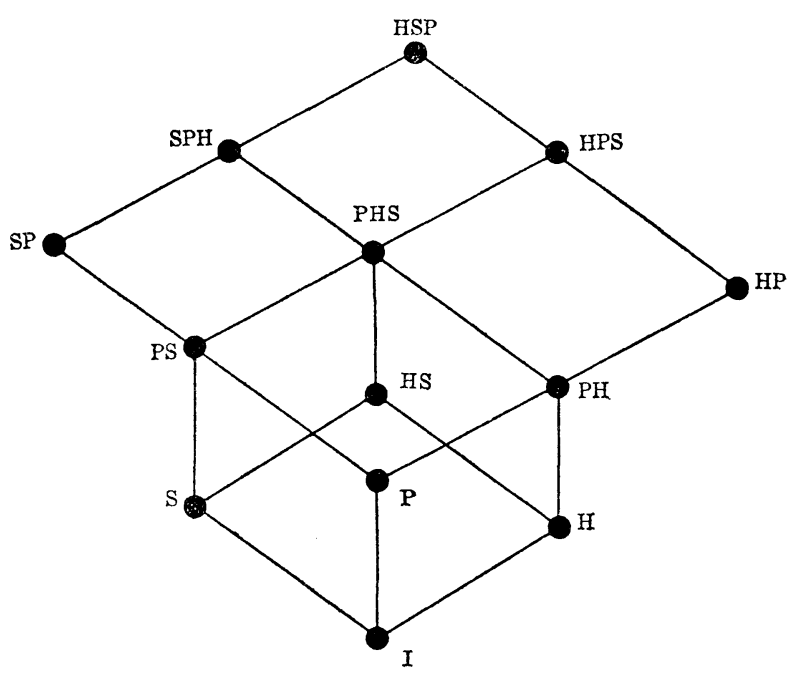

FIGURE 2 
(i) $S \nsupseteq H S$ ，(ii) $H \geqq S P$ ，(iii) $P \geqq H S$ ，(iv) $H P \nsubseteq S P H$, and (v) $S P \geqq$ $H P S$. To see that this will suffice we note that the ordering indicated by the diagram in Figure 2 is consistent with $\leqq$ and that any further collapse in the diagram would contradict one of (i)-(v).

(i) $S \not H P$ : Let $K=\{C\}$ where $C=\{1\} \cup\left\{x_{n}: n=1,2, \cdots\right\}$ and $1>x_{1}>x_{2} \cdots$. Clearly the two-element chain is a subalgebra of $C$ so it is in $S(K)$. Suppose $C_{2} \in H P(K)$. Let $f: \prod_{i \in I} C \rightarrow C_{2}$ be an epimorphism. Suppose $f(x)=0$. Since $C$ has no smallest element, we take $y \in \prod_{i \in I} C$ such that $y_{i}<x_{i}$ for each $i \in I$. We must have $f(y)=0$. Then $(x * y)_{i}=x_{i} * y_{i}=y_{i}$ so $x * y=y$. But this gives $0=$ $f(y)=f(x * y)=f(x) * f(y)=0 * 0=1$ a contradiction.

(ii) $H \not S P$ : Let $K=\{L\}$ where $L=\Pi\left(C_{n} ; n=1,2, \cdots\right\}$. Let $C_{n}=\left\{1, y^{2}, \cdots, y^{n}\right\}$ with $y^{1}>y^{2}>\cdots>y^{n}$. Let

$$
J=\left\{x \in L: x_{i}=1 \text { for all but a finite number of } i \text { 's }\right\} \text {. }
$$

Clearly $J$ is a filter. For $x \in L$ we let $[x]$ denote the equivalence class in $L / J$ which contains $x$. For each $n$ we let $\bar{n}$ be the element of $L$ defined by

$$
\bar{n}_{i}= \begin{cases}y^{n} & \text { if } \quad i \geqq n \\ 1 & \text { if } \quad i<n .\end{cases}
$$

Now if $n>m$, then for any $i \geqq n$ we have $\bar{n}_{i}=y^{n}<y^{m}=\bar{m}_{i}$. Thus $\bar{n}_{i}<\bar{m}_{i}$ for all but a finite number of $i$ 's. Thus $[\bar{n}]<[\bar{m}]$. Also for all but a finite number of $i$ 's we have $\bar{m}_{i} * \bar{n}_{i}=\bar{n}_{i}$ so $[\bar{m}] *[\bar{n}]=[\bar{n}]$. Thus we have an infinite chain $C=\{[\overline{1}],[\overline{2}], \cdots\} \leqq_{s} L / J$. Thus $C \in$ $S H(K)$. Now if $H(K) \subseteq S P(K)$ we get $C \in S H(K) \subseteq S S P(K)=S P(K)$. Since $C$ is subdirectly irreducible, Lemma 2.6 gives $C \in S(K)$. But if $C \leqq{ }_{s} \Pi C_{n}$, we apply Lemma 2.6 again to get $C \leqq_{s} C_{n}$ for some $n$, a contradiction.

(iii) $P \geqq H S$ : Let $K=\left\{C_{2}\right\}$. Then $C_{2} \times C_{2} \in P(K)$, but $C_{2} \times C_{2} \in$ $H S(K)$.

(iv) $H P \leqq S P H$ : If $H P \leqq S P H$, then $S H P \leqq S P H$. We show that this is not the case. Let $K=\left\{C_{n}: n=1,2, \cdots\right\}$. Clearly $H(K)=K$ so $S P H(K)=S P(K)$. Now in (ii) we saw that $S H\left(\Pi C_{n}\right)$ has an infinite subdirectly irreducible member $C$. Thus $C \in S H P(K)$. Now if $C \in$ $S P H(K)$ we have by Lemma 2.6 that $C \in S(K)=K$, but this is not the case. Hence $C \in S H P(K)$, but $C \notin S P H(K)$.

(v) $S P \not H P S$ : Let $K=\left\{C_{2}\right\}$. Let $L$ consist of all cofinite subsets of an infinite set. Then $L \in S P(K)$. We note that $L$ has no least element. It is clear that any member of $H P S(K)$ has a least element. 


\section{REFERENCES}

1. G. Birkhoff, On the structure of abstract algebra, Proc. Cambridge Phil. Soc., 31 (1935), 433-453.

2. Gratzer, Universal Algebra, Van Nostrand (1958).

3. B. Jonsson, Algebras whose congruence lattices are distributive, Math. Scand., 21 (1967), 110-121.

4. A. Monteiro, Axiomes independents pour les algebres de Brouwer, Revista de la Union Mathematica Argentina y de la Association Fisira Argentina, 27 (1955), 149-160.

5. W. Nemitz, Implicative Semi-lattices, Trans. Amer. Math. Soc., 117 (1965), 128-142.

6. - On the lattice of filters of an implicative semilattice, J. Math. Mechanics, 18 (1969), 683-688.

7. - Semi-boolean lattices, Notre Dame J. Formal Logic, 10 (1969), 235-238.

8. D. Pigozzi, On some operations on classes of algebras, Notices Amer. Math. Soc., 13 (1966), 829.

Received June 30, 1970. This research was partially supported by NSF grant GP-3718.

Southwestern at Memphis 



\title{
PACIFIC JOURNAL OF MATHEMATICS
}

\author{
EDITORS
}

\author{
H. SAMelson \\ Stanford University \\ Stanford, California 94305 \\ C. R. HoBBy \\ University of Washington \\ Seattle, Washington 98105
}

J. DugunduI

Department of Mathematics

University of Southern California

Los Angeles, California 90007

RICHARD ARENS

University of California

Los Angeles, California 90024

\section{ASSOCIATE EDITORS}

\author{
E. F. BeCKENBACH
}

B. H. NEUMANN

F. WoLF

K. YOSHIDA

\section{SUPPORTING INSTITUTIONS}

\author{
UNIVERSITY OF BRITISH COLUMBIA \\ CALIFORNIA INSTITUTE OF TECHNOLOGY \\ UNIVERSITY OF CALIFORNIA \\ MONTANA STATE UNIVERSITY \\ UNIVERSITY OF NEVADA \\ NEW MEXICO STATE UNIVERSITY \\ OREGON STATE UNIVERSITY \\ UNIVERSITY OF OREGON \\ OSAKA UNIVERSITY \\ UNIVERSITY OF SOUTHERN CALIFORNIA
}

\author{
STANFORD UNIVERSITY \\ UNIVERSITY OF TOKYO \\ UNIVERSITY OF UTAH \\ WASHINGTON STATE UNIVERSITY \\ UNIVERSITY OF WASHINGTON \\ AMERICAN MATHEMATICAL SOCIETY \\ CHEVRON RESEARCH CORPORATION \\ NAVAL WEAPONS CENTER
}

The Supporting Institutions listed above contribute to the cost of publication of this Journal, but they are not owners or publishers and have no responsibility for its content or policies.

Mathematical papers intended for publication in the Pacific Journal of Mathematics should be in typed form or offset-reproduced, (not dittoed), double spaced with large margins. Underline Greek letters in red, German in green, and script in blue. The first paragraph or two must be capable of being used separately as a synopsis of the entire paper. The editorial "we" must not be used in the synopsis, and items of the bibliography should not be cited there unless absolutely necessary, in which case they must be identified by author and Journal, rather than by item number. Manuscripts, in duplicate if possible, may be sent to any one of the four editors. Please classify according to the scheme of Math. Rev. Index to Vol. 39. All other communications to the editors should be addressed to the managing editor, Richard Arens, University of California, Los Angeles, California, 90024.

50 reprints are provided free for each article; additional copies may be obtained at cost in multiples of 50 .

The Pacific Journal of Mathematics is published monthly. Effective with Volume 16 the price per volume (3 numbers) is $\$ 8.00$; single issues, $\$ 3.00$. Special price for current issues to individual faculty members of supporting institutions and to individual members of the American Mathematical Society: $\$ 4.00$ per volume; single issues $\$ 1.50$. Back numbers are available.

Subscriptions, orders for back numbers, and changes of address should be sent to Pacific Journal of Mathematics, 103 Highland Boulevard, Berkeley, California, 94708.

PUBLISHED BY PACIFIC JOURNAL OF MATHEMATICS, A NON-PROFIT CORPORATION

Printed at Kokusai Bunken Insatsusha (International Academic Printing Co., Ltd.), 7-17, Fujimi 2-chome, Chiyoda-ku, Tokyo, Japan. 


\section{Pacific Journal of Mathematics}

\section{Vol. 37, No. $3 \quad$ March, 1971}

Mohammad Shafqat Ali and Marvin David Marcus, On the degree of the

minimal polynomial of a commutator operator ................ 561

Howard Anton and William J. Pervin, Integration on topological

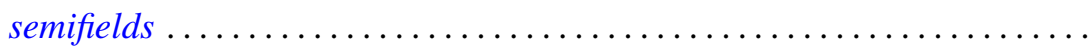

Martin Bartelt, Multipliers and operator algebras on bounded analytic

functions .................................... 575

Donald Earl Bennett, Aposyndetic properties of unicoherent continua ...... 585

James W. Bond, Lie algebras of genus one and genus two ............. 591

Mario Borelli, The cohomology of divisorial varieties ............... 617

Carlos R. Borges, How to recognize homeomorphisms and isometries ....... 625

J. C. Breckenridge, Burkill-Cesari integrals of quasi additive interval

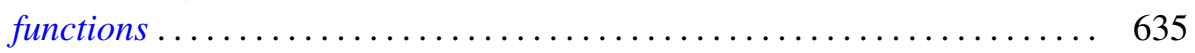

J. Csima, A class of counterexamples on permanents ................ 655

Carl Hanson Fitzgerald, Conformal mappings onto $\omega$-swirly domains . . . . . . 657

Newcomb Greenleaf, Analytic sheaves on Klein surfaces .............. 671

G. Goss and Giovanni Viglino, C-compact and functionally compact

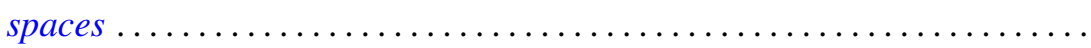

Charles Lemuel Hagopian, Arcwise connectivity of semi-aposyndetic plane

continua ..................................... 683

John Harris and Olga Higgins, Prime generators with parabolic limits ...

David Michael Henry, Stratifiable spaces, semi-stratifiable spaces, and their

relation through mappings .......................

Raymond D. Holmes, On contractive semigroups of mappings ........... 701

Joseph Edmund Kist and P. H. Maserick, BV-functions on semilattices ....... 711

Shûichirô Maeda, On point-free parallelism and Wilcox lattices ........... 725

Gary L. Musser, Linear semiprime $(p ; q)$ radicals ................. 749

William Charles Nemitz and Thomas Paul Whaley, Varieties of implicative

semilattices..................................... 759

Jaroslav Nešetřil, A congruence theorem for asymmetric trees ............ 771

Robert Anthony Nowlan, A study of $H$-spaces via left translations .......... 779

Gert Kjærgaard Pedersen, Atomic and diffuse functionals on a $C^{*}$-algebra ... 795

Tilak Raj Prabhakar, On the other set of the biorthogonal polynomials

suggested by the Laguerre polynomials...

801

Leland Edward Rogers, Mutually aposyndetic products of chainable

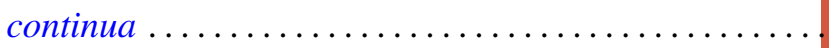

Frederick Stern, An estimate for Wiener integrals connected with squared

error in a Fourier series approximation.

Leonard Paul Sternbach, On k-shrinking and k-boundedly complete basic

sequences and quasi-reflexive spaces .................... 817

Pak-Ken Wong, Modular annihilator $A^{*}$-algebras ........ 